\title{
Intelligent control of mode-locked femtosecond pulses by time-stretch-assisted real-time spectral analysis
}

\author{
Guoqing Pu', Lilin Yi', Li Zhang ${ }^{1}$, Chao Luo ${ }^{1}$, Zhaohui $\mathrm{Li}^{2}$ and Weisheng $\mathrm{Hu}^{1}$
}

\begin{abstract}
Mode-locked fiber lasers based on nonlinear polarization evolution can generate femtosecond pulses with different pulse widths and rich spectral distributions for versatile applications through polarization tuning. However, a precise and repeatable location of a specific pulsation regime is extremely challenging. Here, by using fast spectral analysis based on a time-stretched dispersion Fourier transform as the spectral discrimination criterion, along with an intelligent polarization search algorithm, for the first time, we achieved real-time control of the spectral width and shape of mode-locked femtosecond pulses; the spectral width can be tuned from 10 to $40 \mathrm{~nm}$ with a resolution of $\sim 1.47 \mathrm{~nm}$, and the spectral shape can be programmed to be hyperbolic secant or triangular. Furthermore, we reveal the complex, repeatable transition dynamics of the spectrum broadening of femtosecond pulses, including five middle phases, which provides deep insight into ultrashort pulse formation that cannot be observed with traditional mode-locked lasers.
\end{abstract}

\section{Introduction}

Because pulse trains achieve excellent performance with a simple laser setup ${ }^{1}$, passively mode-locked fiber lasers (MLFLs) based on nonlinear polarization evolution (NPE) have numerous applications, ranging from highresolution atomic clocks ${ }^{2,3}$ to optical frequency measurements ${ }^{4,5}$, photonic analog-to-digital converters $(\mathrm{ADCs})^{6}$, photonic radars ${ }^{7}$, fine ranging metrology $\mathrm{y}^{8,9}$, and astronomy $^{10,11}$. However, NPE-based MLFLs are difficult to operate in the desired pulsation regime via manual polarization tuning and are prone to detaching from the desired regime due to polarization drift from environmental disturbances ${ }^{12}$.

To address these challenges, automatic ${ }^{13-17}$ or intelligent $^{18}$ mode-locking techniques using adaptive algorithms and electric polarization controllers (EPCs) have

\footnotetext{
Correspondence: Lilin Yi (lilinyi@sjtu.edu.cn)

${ }^{1}$ State Key Lab of Advanced Communication Systems and Networks, Shanghai Institute for Advanced Communication and Data Science, Shanghai Jiao Tong University, Shanghai 200240, China

${ }^{2}$ Sun Yat-sen University/Southern Marine Science and Engineering Guangdong Laboratory, Zhuhai, China
}

emerged in recent years. Several automatic mode-locking lasers use temporal information to help identify the mode-locking regimes ${ }^{14,17,18}$. Combined with automatic optimization algorithms, such lasers can successfully reach the mode-locking regimes, but their pulse width and spectral shape are unpredictable. Thus, automatic mode-locking techniques based on a temporal discrimination alone cannot achieve mode-locking with the possible shortest pulse width and desired spectral distribution. Even though optical spectral information can be utilized in automatic mode-locking using an optical spectrum analyzer $(\mathrm{OSA})^{14-16}$, such bulky and slow equipment only obtains integrated spectral information and therefore cannot be used for real-time mode-locking. A time-stretch dispersion Fourier transform (TS-DFT) produces a map between the spectrum and the temporal pulse by using a dispersive medium ${ }^{19}$. Combined with a real-time oscilloscope, a TS-DFT operates as a real-time OSA, resolving the issue of the low frame rate in a traditional $\mathrm{OSA}^{20}$. In numerous works, TS-DFT-based fast spectral analysis has been used with mode-locked lasers to observe soliton dynamics, including soliton explosions ${ }^{21,22}$,

\section{(c) The Author(s) 2020}

(c) (i) Open Access This article is licensed under a Creative Commons Attribution 4.0 International License, which permits use, sharing, adaptation, distribution and reproduction cc in any medium or format, as long as you give appropriate credit to the original author(s) and the source, provide a link to the Creative Commons license, and indicate if changes were made. The images or other third party material in this article are included in the article's Creative Commons license, unless indicated otherwise in a credit line to the material. If material is not included in the article's Creative Commons license and your intended use is not permitted by statutory regulation or exceeds the permitted use, you will need to obtain permission directly from the copyright holder. To view a copy of this license, visit http://creativecommons.org/licenses/by/4.0/. 
build-up processes of various pulsation regimes ${ }^{23-25}$, dissipative solitons ${ }^{26}$, soliton molecules ${ }^{27-29}$, sophisticated soliton dynamics ${ }^{30-34}$, and the transition dynamics between pulsation regimes ${ }^{35,36}$. In this article, for the first time, we propose using TS-DFT-based fast spectral analysis as the discrimination criterion to achieve rich modelocking regimes. By simply inserting a dispersion medium into the real-time feedback loop of an automatic modelocking laser and combining this method with an intelligent polarization search using a genetic algorithm (GA), we can manipulate the spectral width and shape of the mode-locked femtosecond pulses in real time. The spectral width of the mode-locked femtosecond pulses can be tuned from 10 to $40 \mathrm{~nm}$ with a resolution of $\sim 1.47 \mathrm{~nm}$, and the spectral shape can be programmed to be hyperbolic secant or triangular. With real-time control of the spectral width and shape of the mode-locking pulses, we reveal the complex and repeatable transition dynamics from the narrow-spectrum mode-locking regime to the widespectrum mode-locking regime, including five middle phases: a relaxation oscillation (RO), single soliton state, multi-soliton state, triangle-spectrum transition, and chaotic transition, providing deep insight into the ultrashort pulse formation that cannot be observed with traditional mode-locked lasers. We believe our proposed lowcost, portable, spectrum-programmable intelligent modelocking fiber laser will find widespread applications in both research and industry.

\section{Results}

Principles of the time-stretch-assisted real-time pulse controller (TSRPC)

Figure 1 shows the experimental setup of the intelligent mode-locking fiber laser with an embedded (TSRPC. The laser cavity length is $\sim 54.7 \mathrm{~m}$, corresponding to a fundamental repetition rate of $\sim 3.78 \mathrm{MHz}$. An 8 -m erbiumdoped fiber (EDF) with a dispersion of $-38 \mathrm{ps}(\mathrm{nm} \mathrm{km})^{-1}$, serving as the gain medium, was pumped by a $980-\mathrm{nm}$ laser through a wavelength division multiplexer (WDM). Considering the trade-off between the precision of the spectral control and the low sampling rate of the ADC, we inserted a 30- $\mathrm{m}$ single-mode fiber (SMF) after the EDF to reduce the fundamental repetition rate. An optical coupler retains most of the power inside the cavity for the oscillation, and the rest of the power is sent out for the feedback and measurement. The isolator ensures that the laser cavity operates only in the clockwise direction. The $\mathrm{EPC}$, driven by four DC voltage channels, can generate all possible polarization states over the Poincaré sphere, where each set of voltages corresponds to a specific polarization state and can respond on a timescale of microseconds (see the "Materials and methods" section). The polarizer is the core component combining the EPC to produce artificial saturable absorption in NPE-based mode-locking. The laser is a soliton laser ${ }^{37}$ with a net cavity dispersion of $\sim 8.96 \mathrm{ps}(\mathrm{nm} \mathrm{km})^{-1}$. Part of the output power is sent to the measurement system (see the "Materials and methods" section) for characterization, and the rest is used for feedback. The lower part of Fig. 1 shows the TSRPC for the MLFLs, including a dispersion compensation fiber (DCF), a photodiode (PD), and a GAbased intelligent real-time controller (see the "Materials and methods" section). The DCF with a dispersion parameter of $-1696 \mathrm{ps} \mathrm{nm}^{-1}$ in the $\mathrm{C}$ band is used to complete the temporal-to-spectral mapping. Thus, the theoretical precision of the spectral control, determined by both the sampling interval of the ADC and the dispersion of the DCF, is $\sim 1.47 \mathrm{~nm}$.

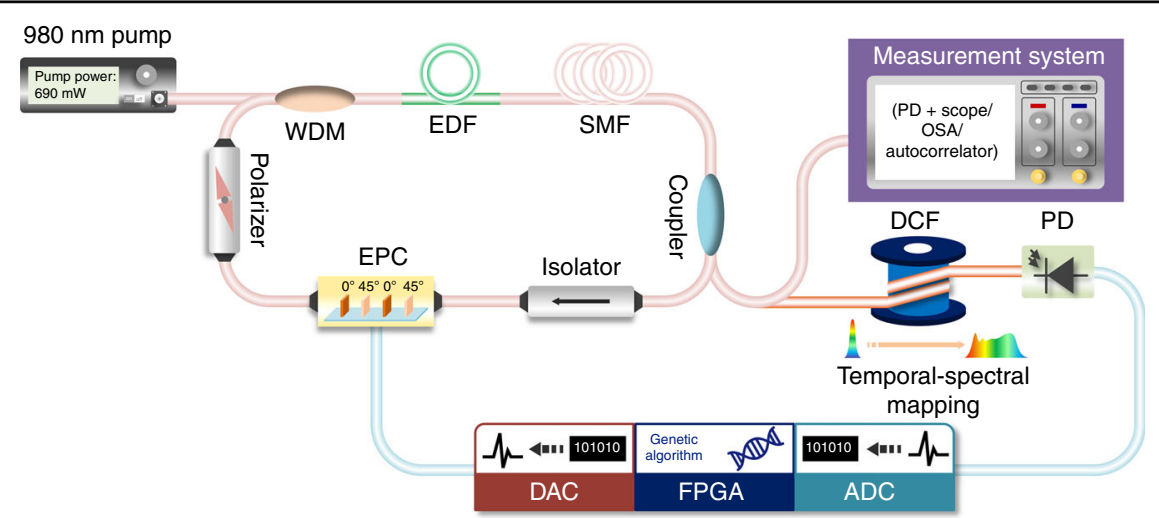

Fig. 1 Experimental setup. The intelligent mode-locking fiber laser with an embedded time-stretch-assisted real-time pulse controller (TSRPC). The electric polarization controller (EPC) and the polarizer together produce artificially saturated absorption in nonlinear polarization evolution (NPE)based mode locking. Part of the output power is sent to the measurement system for characterization, and the rest is used for feedback. The TSRPC consists of a dispersion compensation fiber (DCF) to complete the temporal-spectral mapping, a photodiode (PD), and a genetic algorithm-based intelligent real-time optimizer. 


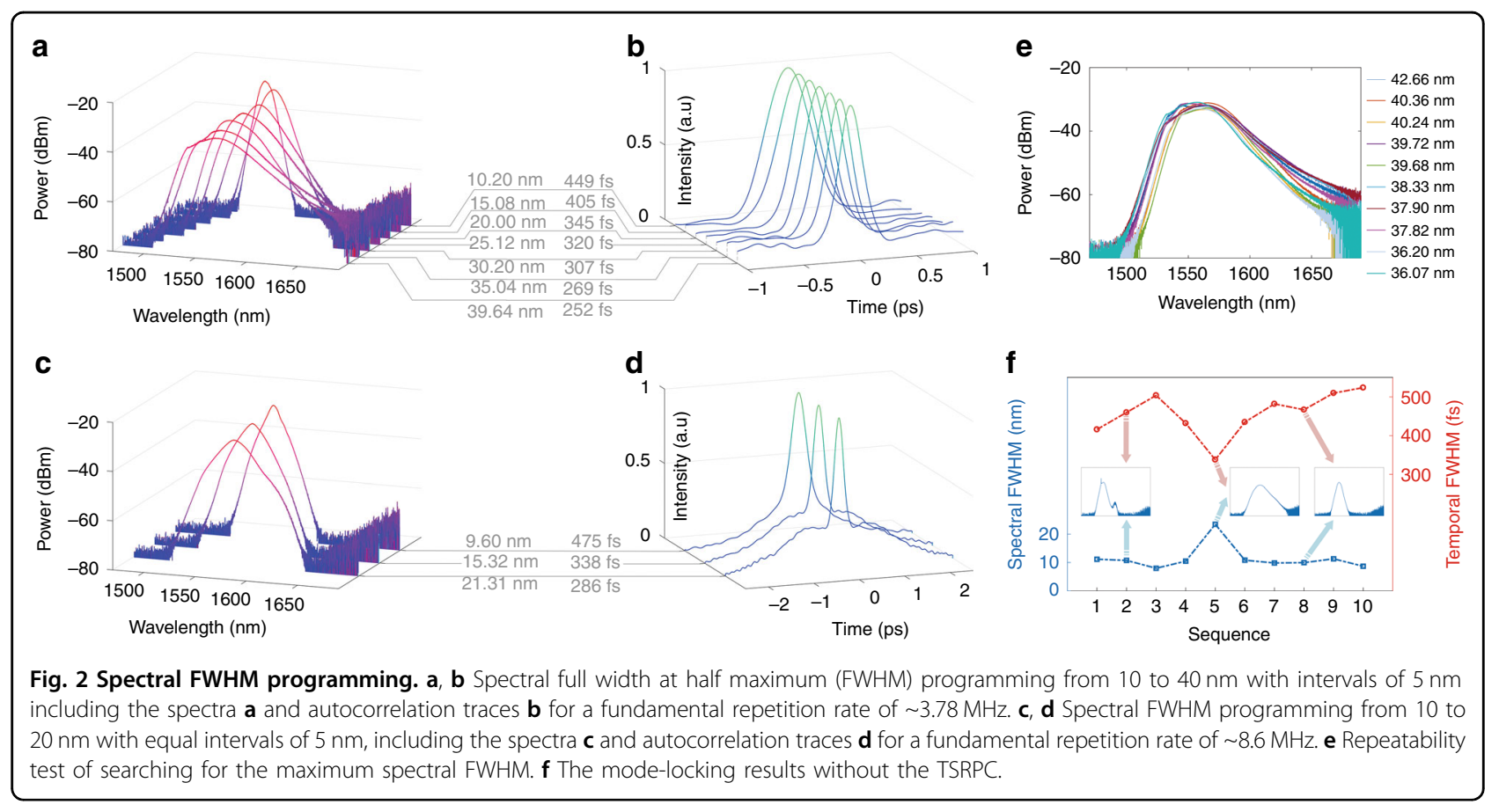

The core of the TSRPC is the intelligent real-time optimizer based on the GA, a global optimization algorithm inspired by species reproduction ${ }^{38}$. First, the GA randomly generates the initial generation. Each individual in the generation has four genes, which correspond to the four control voltages of the EPC. Then, the fitness of each individual is calculated by extracting pulses from the dispersed consecutive waveform through the slope. The fitness functions change based on the spectral shaping target. For example, the fitness function for spectral full width at half maximum (FWHM) programming is the spectral width of the current pulse, while the function for spectral shape programming is the normalized meansquared error (NMSE) between the current pulse and the desired pulse. This method is different from traditional GA-based automatic mode-locking ${ }^{13-16}$, where the algorithm iterates through all the given iterations and then artificially checks if mode-locking is achieved via off-line measurements. Here, we add spectral discrimination (see the "Materials and methods" section) based on fast spectral analysis into the algorithm to judge whether the current spectrum reaches the desired target. The algorithm exits immediately when the desired spectrum is achieved, greatly reducing the algorithm run time by avoiding the remaining calculations (see the "Materials and methods" section). Then, standard genetic operations -including roulette selection, cross, mutation, and reinsertion-are performed to breed the next generation. The GA strives to understand the mapping between the polarization and fitness by iterating and then searches for the polarization with the best fitness.

\section{Spectral FWHM programming}

With the TSRPC, the output spectral FWHM can be programmed. A certain FWHM range is set according to the target FWHM to precisely tune the spectral FWHM. For example, if the target spectral FWHM is $15 \mathrm{~nm}$, the pre-set range is $14-16 \mathrm{~nm}$. The spectrum with the target FWHM is found when the fitness of an individual falls into the pre-set range. Figure 2 shows the experimental results of the spectral FWHM programming, assessing a series of target FWHMs from 10 to $40 \mathrm{~nm}$ with an interval of $5 \mathrm{~nm}$. The programming results are shown in Fig. 2a. The seven spectral FWHMs shown in Fig. 2a are quite near the pre-set target FWHM, indicating the validity of the TSRPC in programming the spectral FWHM. Figure $2 \mathrm{~b}$ shows the corresponding autocorrelation trace of these spectra. This result agrees with the theory that the temporal duration decreases as the spectral width increases. Thus, the temporal duration of ultrashort pulses can also be programmed to a certain degree by using the TSRPC. Note that the measured shortest pulse width is only $252 \mathrm{fs}$ because we have not optimized the cavity dispersion and because an optical amplifier is used before the autocorrelator, which may broaden the pulse width. To test the generalization of the TSRPC, we remove the 30-m SMF inserted after the EDF in the laser cavity, increasing the fundamental repetition rate to $\sim 8.6 \mathrm{MHz}$. Now, the laser operates as a stretched-pulse laser since the net cavity dispersion reduces to approximately $-1.26 \mathrm{ps}(\mathrm{nm} \mathrm{km})^{-1}$, which is a near-zero dispersion value $^{37}$. Although the maximum spectral width is reduced by half to $\sim 20 \mathrm{~nm}$, the experimental results in Fig. 2c, d 


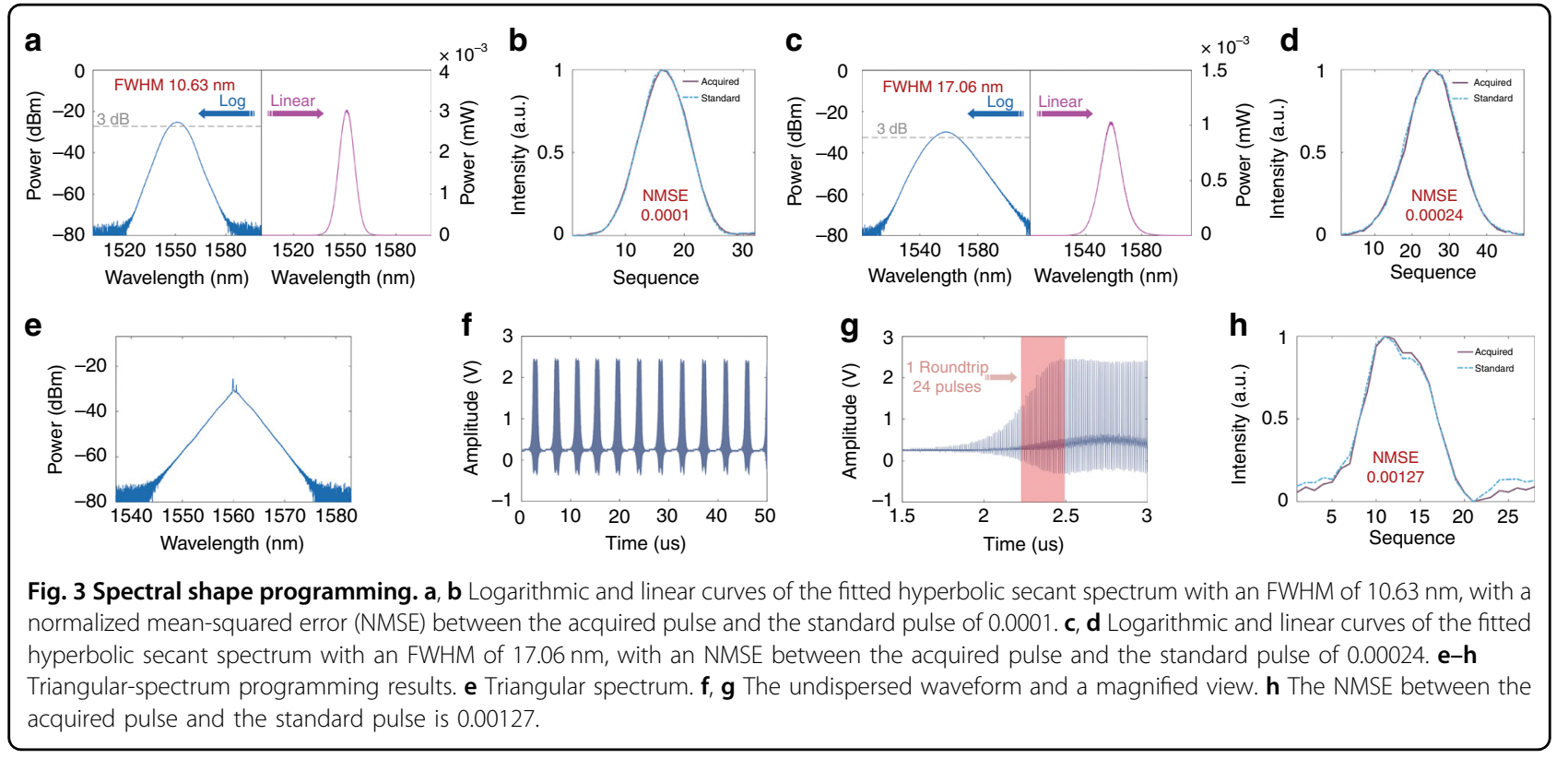

reveal a concordant conclusion, further validating the TSRPC in programming the spectral width. Intriguingly, as the spectrum widens, the probability of the appearance of continuous-wave components decreases. Figure $2 \mathrm{e}$ shows multiple results of searching for the maximum spectral width at a fundamental repetition rate of $3.78 \mathrm{MHz}$. While searching for the maximum spectral width, there is no upper limit, and the lower limit is constantly adjusted in numerous trials until an appropriate lower limit for the maximum spectral width is found. The spectra in Fig. 2e show the same longer tail on the right side, even though the spectral width varies from 36.07 to $42.66 \mathrm{~nm}$. This result demonstrates that this system is repeatable to some extent. In contrast, using our previous intelligent mode-locking method ${ }^{18}$ without the TSRPC, we conduct 10 consecutive measurements of the spectral FWHM and temporal FWHM of the final fundamental mode-locking regime, and the results are shown in Fig. 2f. The output spectral widths and the temporal durations are uncontrollable, as shown in Fig. 2f. The spectral widths are distributed around $10 \mathrm{~nm}$, because a spectrum with an FWHM of $\sim 10 \mathrm{~nm}$ is the most common fundamental mode-locking regime that corresponds to a large polarization solution space in this laser. In addition, the spectral shapes are not controllable, as shown in the three insets of Fig. 2f, and the possibility of the output spectra exhibiting strong continuous-wave components is larger than that for the TSRPC method according to experimental experience. For instance, the strong continuous-wave components in the spectrum shown in the left inset of Fig. $2 \mathrm{f}$ signify a non-ideal mode-locking regime.

\section{Spectral shape programming}

The TSRPC can also be used to fit various spectral shapes. First, a dispersed standard pulse carrying spectral information is required, and different objective spectral shapes have different standard pulses. A set of experimental data (i.e., the dispersed temporal waveform) corresponding to the objective spectral shape is required to acquire the standard pulse. Notably, the fitness function in shape programming becomes the NMSE between the current pulse and the standard pulse. Then, a threshold is set as the upper limit of the NMSE. The objective spectral shape is successfully fitted when the NMSE between the current pulse and the standard pulse is lower than a preset threshold.

Figure 3 shows the shape programming results for a fundamental repetition rate of $3.78 \mathrm{MHz}$. The upper part shows hyperbolic secant spectra programming with various standard deviations. Figure 3a shows the logarithmic and linear curves of the fitted hyperbolic secant spectrum with an FWHM of $10.63 \mathrm{~nm}$. Figure 3b compares the acquired pulse after fitting with the standard pulse, showing an NMSE of only 0.0001 , a nearly perfect fit. Likewise, Fig. 3c shows the logarithmic and linear curves of the fitted hyperbolic secant spectrum, with a larger FWHM of $17.06 \mathrm{~nm}$. Figure 3d shows an NMSE of just 0.00024 , which is a rather good fit. Notably, the spectral FWHM of the fitted hyperbolic secant spectrum fluctuates within $2 \mathrm{~nm}$ over multiple runs. According to experimental experience, an NMSE lower than $\sim 0.01$ usually represents a good fit. For various spectral shapes, the NMSE equivalently reveals the disparities between the current pulse and the standard pulse. Additionally, a 
triangular spectrum appears for the laser cavity. After extracting the standard pulse from the dispersed waveform, the triangular spectrum is reproduced by fitting the shape using the TSRPC, as shown in Fig. 3e. Figure $3 \mathrm{f}$ shows the undispersed waveform, and Fig. $3 g$ shows a magnified view of the undispersed waveform. The triangular-spectrum regime corresponds to Q-switched mode-locking (QML) because its temporal waveform corresponds to the harmonic mode-locking regime modulated by low-frequency components. Thus, the triangular-spectrum regime is analogous to the breathing behavior in dissipative soliton dynamics ${ }^{31}$. However, the undispersed temporal envelope of the triangularspectrum regime is trapezoidal rather than sinusoidal, as in the ordinary QML regime. There are 24 pulses within a single roundtrip of $\sim 264.6 \mathrm{~ns}$. The number of pulses within a single roundtrip is uncertain and can vary from 3 to 24 during the experiment, even when using the same standard pulse of the triangular spectrum. A comparison between the acquired pulse after fitting and the standard pulse during triangular-spectrum programming is shown in Fig. 3h. An NMSE of 0.00127 signifies a good fit. Theoretically, the output spectrum can be programmed using the TSRPC to be any spectral shape (including soliton bound states ${ }^{27-29}$ ) existing in the laser cavity but only if an $\mathrm{ADC}$ with a higher sampling rate or larger dispersion is used to improve the spectral resolution. Furthermore, combined with a coherent time-stretch transformation $^{39-42}$, the phase information could be acquired, thus providing the possibility to control the phase of the pulses.

\section{Transition dynamics of the spectral width optimization process}

Through the TS-DFT, more dynamics in MLFLs have come to light in recent studies ${ }^{21-35}$, deepening the understanding of the internal dynamics of mode-locking pulse formation and assisting the design of MLFLs. The starting dynamics, from the initial noise fluctuations to various regimes, have been intensively reported ${ }^{23-29}$, but the regime transition dynamics, which are essential processes in mode-locked lasers, are poorly understood. The transition dynamics from Q-switching to mode-locking in a soliton laser have been revealed ${ }^{34}$, and the transition dynamics of the Q-switching, fundamental mode-locking, and harmonic mode-locking regimes ${ }^{35}$ have also been observed recently. For NPE-based MLFLs, their modelocking regimes fall into a large polarization space of different spectral widths and shapes. The evolution from the narrow-spectrum mode-locking regime to the widespectrum mode-locking regime is an essential physical process in producing ultrashort femtosecond pulses, but the corresponding transition dynamics have never been revealed because the physical process is very difficult to control and track in traditional mode-locking lasers. By using the TSRPC to control the spectral width and shape of the mode-locked pulses in real time, however, we revealed the transition dynamics from the narrowspectrum mode-locking regime to the wide-spectrum mode-locking regime and from the triangular-spectrum QML regime (see Supplementary Information) to the wide-spectrum mode-locking regime by recording several sets of experienced voltages of the EPC that led to the different regimes. All of the transitions were produced at a fundamental repetition rate of $3.78 \mathrm{MHz}$, signifying a roundtrip of $\sim 264.6 \mathrm{~ns}$.

We investigated the transition dynamics from the narrow-spectrum mode-locking regime to the widespectrum mode-locking regime, as shown in Fig. 4. The initial spectral FWHM of the narrow-spectrum modelocking regime and the ultimate spectral FWHM of the wide-spectrum mode-locking regime were 15.76 and $31.04 \mathrm{~nm}$, respectively. The entire transition contains five middle phases, which are sequentially the $\mathrm{RO}$, singlesoliton state, multi-soliton state, transient triangularspectrum transition, and chaotic transition including the QML oscillations and the unstable noisy wide-spectrum transition, as shown in Fig. 4a. Figure 4b shows the RO induced by the polarization assignment of the EPC. After the $\mathrm{RO}$ dies out, the laser enters a short-lived singlesoliton state with a noisy background caused by the residual RO. Then, two solitons grow on the noisy background, forming the multi-soliton state. The solitons are generated through the $\mathrm{RO}$, as previously demonstrated $^{23,29}$. The multi-soliton state has three nearly isometric solitons in a single roundtrip, as shown on the left side of Fig. 4c. It is obvious that the middle soliton has the maximum power. One possible reason for this result is that the soliton with the maximum power corresponds to a spectral range that is inside the spectral range of the narrow-spectrum regime. Therefore, more power is preserved, forming the strongest soliton. Another reason might be that the spectral range of the strongest soliton is near the center wavelength of the ultimate wide-spectrum regime. More power is required for wavelengths inside the spectral range of the ultimate regime to complete the transition. The situation in Fig. S1 also supports these two reasons (see Supplementary Information). However, for the multi-soliton state, the temporal amplitude constantly increases due to spectral narrowing of some solitons, such as the middle soliton of the multi-soliton state in Fig. 4a. Thus, the multi-soliton state becomes unstable due to the stronger temporal amplitude and environmental disturbances.

After maintaining the multi-soliton state for $\sim 2000$ roundtrips, the temporal amplitude finally starts fluctuating, and the laser evolves into the triangular-spectrum transition of three pulses in a single roundtrip. The 


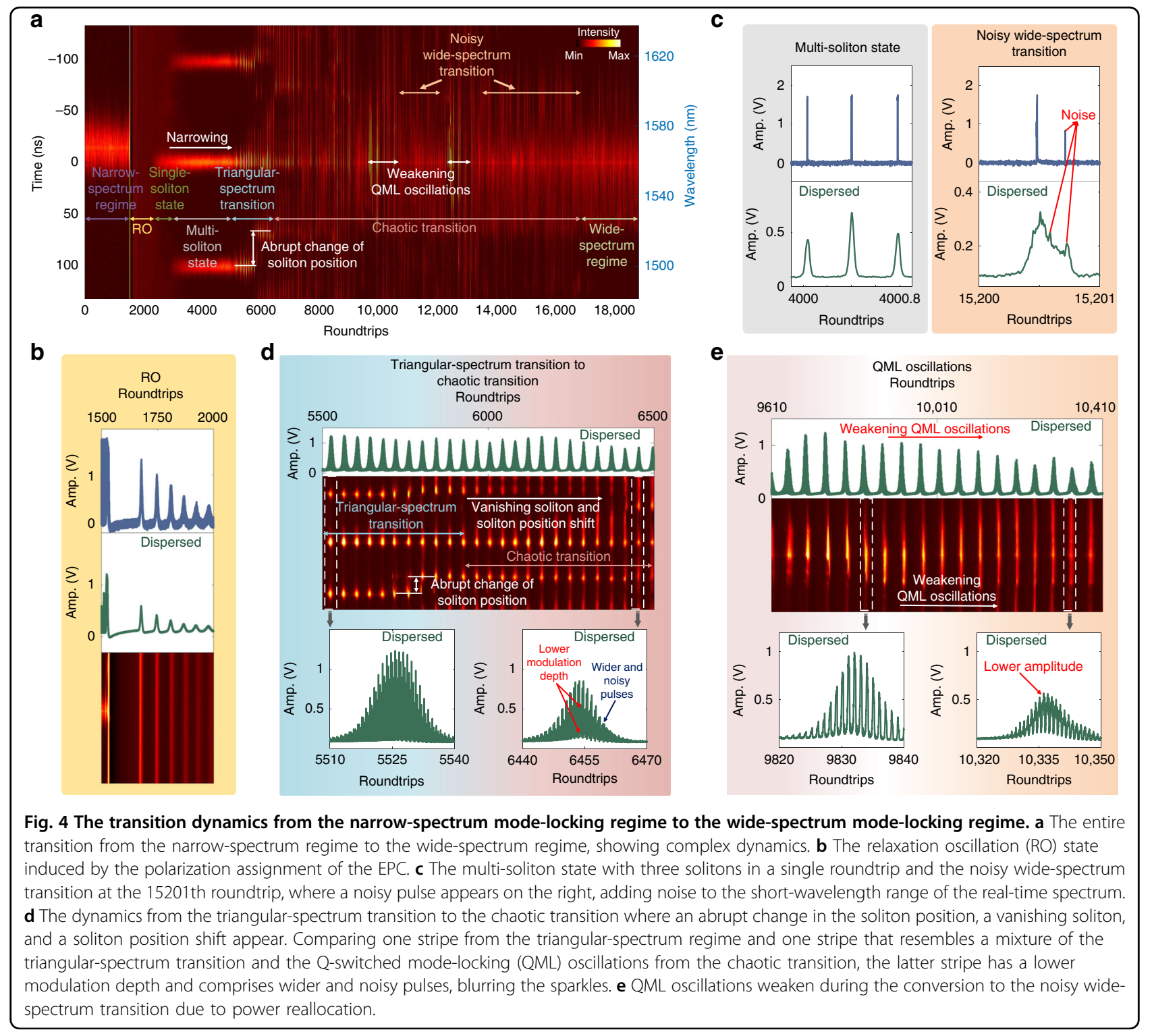

instability originating from the increasing temporal amplitude does not cease and pushes the laser further into the chaotic transition, as shown in Fig. 4d. The soliton changes position abruptly around the 5900th roundtrip inside the triangular-spectrum transition (see Supplementary Information), revealing the complexity of the transition dynamics. Afterwards, both the upper soliton and lower soliton vanish, and the upper soliton simultaneously changes position. Soliton decay and position shift have also appeared in transient dissipative soliton dynamics $^{31}$.

For a comparison, two stripes selected from the triangular-spectrum transition and the chaotic transition are shown at the bottom of Fig. 4d. The latter stripe, with three blurred sparkles, looks like a mixture of the triangular-spectrum transition and the QML oscillations, as shown in Fig. 4e. Different from the RO, the interferogram of Fig. 4e shows an obvious sparkle in each envelope due to the pulses on the envelope. The QML oscillations are also unlike the triangular-spectrum regime because there is only one pulse in a single roundtrip in the QML oscillations. Comparing the two dispersed waveforms, the latter stripe has a lower modulation depth and comprises wider and noisy pulses, blurring the sparkles. The mixture of the triangular-spectrum transition and the QML oscillations holds for nearly 3000 roundtrips in the chaotic transition until it completely turns into QML oscillations. The QML oscillations, which reallocate power, evidently weaken during the conversion to the noisy wide-spectrum transition, as shown in Fig. 4e. The result of the power reallocation-the noisy wide-spectrum transition, in this case-is primarily determined by the 
status of the laser cavity, that is, the polarization. Before entering the noisy wide-spectrum transition, the weakened QML oscillations have a lower amplitude and far noisier pulses than the stronger QML oscillations induced by the power reallocation, as shown at the bottom of Fig. 4e. However, due to internal instabilities (i.e., nonlinear effects) and environmental disturbances, the power reallocation-the QML oscillations-can repeatedly emerge until the spectrum ultimately settles down, as shown in Fig. 4a. Through two rounds of power reallocation, the laser sequentially enters the noisy wide-spectrum transition and remains there until the ultimate wide-spectrum regime dominates. The noisy wide-spectrum transition, shown on the right side of Fig. 4c, has a noise pulse on the right side, providing considerable noise in the shortwavelength range of the real-time spectrum.

Furthermore, the transition from the triangularspectrum regime to the wide-spectrum mode-locking regime, determined by the same set of EPC controlling voltages (i.e., the terminal polarization), shows dynamics analogous to the transition in Fig. 4 (see Supplementary Information). In conclusion, the transition dynamics depend considerably on the concrete objective regime, which is controlled by the terminal polarization in the experiments. We conducted multiple observations with the same original and terminal polarization states, and although no two transitions showed identical dynamics, all the transitions showed nearly the same middle phases, demonstrating that the transition dynamics observations were repeatable.

\section{Discussion}

Using TS-DFT-based fast spectral analysis, we demonstrated the TSRPC method, which helps NPE-based MLFLs spectrally program the output pulse train. Benefitting from the TS-DFT and the real-time GA optimizer, the TSRPC overcomes the considerable slowness, cost, and bulkiness of traditional OSAs used in previous automatic mode-locking lasers ${ }^{14-16}$. The TSRPC can be made even more portable by replacing the DCF with a small optical grating, and its spectral programming resolution can be improved by using an ADC with a higher sampling rate or a medium with large dispersion. Moreover, we revealed the transitions from the narrowspectrum mode-locking regime and from the triangularspectrum QML regime (see Supplementary Information) to the wide-spectrum mode-locking regime with intriguing and complex dynamics. These results showed that the transition dynamics are mostly determined by the terminal polarization, regardless of the original polarization. The TSRPC, which can program the spectral FWHM and spectral shape, greatly broadens the applications of NPE-based MLFLs and undoubtedly contributes to NPEbased MLFLs.

\section{Materials and methods The EPC}

Inside the EPC, there are four phase retarders, and their optical axes are oriented at $0^{\circ}$ and $45^{\circ}$. Each phase retarder is individually controlled through a DC voltage and can induce a phase delay of 0 to $\pi$ radians almost continuously. Hence, each phase retarder functions as a variable waveplate. The light is coupled into and out of the EPC through two collimators.

\section{Experimental setup}

An ADC, a field-programmable gate array (FPGA), and four digital-to-analog converters (DACs) controlling the four channels of the EPC constitute the GA-based intelligent real-time controller. The 400-MSa s${ }^{-1} \mathrm{ADC}$ in the GA-based real-time optimizer has an 8-bit resolution and a maximum input amplitude of $1.2 \mathrm{~V}_{\mathrm{pp}}$. Four $100-\mathrm{MSa} \mathrm{s}^{-1}$ DACs with 12-bit resolution control the four channels of the EPC. Thus, the size of the polarization space reaches $4096^{4}$. The instrument used to characterize the temporal and spectral features has three parts: to characterize the temporal features, a $10-\mathrm{GHz} \mathrm{PD}$ and a real-time oscilloscope with a sampling rate of $2.5 \mathrm{GSa} \mathrm{s}^{-1}$ capture the temporal waveforms. The temporal duration of the output pulses is measured by an auto-correlator, and the spectra of the output pulses are measured using a traditional OSA.

A high-speed real-time oscilloscope with a sampling rate of $10 \mathrm{GSa} \mathrm{s}{ }^{-1}$ measures both the undispersed and dispersed data in the transition observations, producing a spectral resolution of $\sim 0.06 \mathrm{~nm}$. Additionally, the data acquisition of the transition observations is ensured by the trigger scheme achieved via the FPGA. When the EPC is assigned a set of new voltages, the FPGA will send out a trigger signal to inform the oscilloscope that the transition has begun.

\section{Spectral discrimination in the GA}

For both spectral FWHM programming and spectral shape programming, the fitness of each individual (i.e., each polarization or each set of DAC output voltages) inside the GA is not determined by the fitness of a single stretched pulse (i.e., a single real-time spectrum) but is determined by the mean fitness of several stretched pulses that are sampled at different times. The aim behind this choice is to improve the accuracy when assessing each polarization. When the mean fitness surpasses the pre-set threshold for the first time, the spectrum has not yet been considered to be one of the target spectra. The algorithm is then delayed for several seconds. Then, a new pulse train is sampled by the ADC, and a second check is performed to ensure that most of the various transient instabilities ${ }^{43}$ can be filtered. When the fitness calculated from the new pulse train passes the second check (i.e., surpassing the pre-set threshold), the spectrum is finally considered to be one of the target 
spectra, and the current polarization is considered to be one solution. After successfully searching one solution, the algorithm terminates, thereby skipping the calculation of the remaining generations in the middle of the GA optimization to reduce the algorithm running time.

\section{Acknowledgements}

This work is supported by the National Natural Science Foundation of China (61575122).

\section{Author contributions}

L.Y. conceived the idea. G.P. designed and performed the experiments under the supervision of L.Y. L.Z. and C.L. assisted in the experiments. Z.L. assisted in setting up the TS-DFT measurement system. All authors discussed the results and contributed to writing the manuscript.

\section{Conflict of interest}

The authors declare that they have no conflict of interest.

Supplementary information is available for this paper at https:/doi.org/ 10.1038/s41377-020-0251-x.

Received: 7 November 2019 Revised: 9 January 2020 Accepted: 14 January 2020

Published online: 28 January 2020

\section{References}

1. Kim, J. \& Song, Y. Ultralow-noise mode-locked fiber lasers and frequency combs: principles, status, and applications. Adv. Opt. Photonics 8, 465-540 (2016).

2. Bloom, B. J. et al. An optical lattice clock with accuracy and stability at the $10^{-18}$ level. Nature 506, 71-75 (2014).

3. Nemitz, N. et al. Frequency ratio of $\mathrm{Yb}$ and Sr clocks with $5 \times 10^{-17}$ uncertainty at 150 seconds averaging time. Nat. Photonics 10, 258-261 (2016).

4. Udem, T., Holzwarth, R. \& Hänsch, T. W. Optical frequency metrology. Nature 416, 233-237 (2002).

5. Diddams, S. A. et al. Direct link between microwave and optical frequencies with a $300 \mathrm{THz}$ femtosecond laser comb. Phys. Rev. Lett. 84, 5102-5105 (2000).

6. Khilo, A. et al. Photonic ADC: overcoming the bottleneck of electronic jitter. Opt. Express 20, 4454-4469 (2012).

7. Ghelfi, P. et al. A fully photonics-based coherent radar system. Nature 507, 341-345 (2014).

8. Lee, J. et al. Time-of-flight measurement with femtosecond light pulses. Nat. Photonics 4, 716-720 (2010).

9. Coddington, I., Swann, W. C., Nenadovic, L. \& Newbury, N. R. Rapid and precise absolute distance measurements at long range. Nat. Photonics 3, 351-356 (2009).

10. Li, C. H. et al. A laser frequency comb that enables radial velocity measurements with a precision of $1 \mathrm{~cm} \mathrm{~s}^{-1}$. Nature $\mathbf{4 5 2}, 610-612$ (2008).

11. Steinmetz, T. et al. Laser frequency combs for astronomical observations. Science 321, 1335-1337 (2008).

12. Karlsson, O., Brentel, J. \& Andrekson, P. A. Long-term measurement of PMD and polarization drift in installed fibers. J. Lightwave Technol. 18, 941-951 (2000).

13. Andral, U. et al. Fiber laser mode locked through an evolutionary algorithm. Optica 2, 275-278 (2015)

14. Woodward, R. I. \& Kelleher, E. J. R. Towards 'smart lasers': self-optimisation of an ultrafast pulse source using a genetic algorithm. Sci. Rep. 6, 37616 (2016).

15. Woodward, R. I. \& Kelleher, E. J. R. Genetic algorithm-based control of birefringent filtering for self-tuning, self-pulsing fiber lasers. Opt. Lett. 42 2952-2955 (2017)
16. Winters, D. G. et al. Electronic initiation and optimization of nonlinear polarization evolution mode-locking in a fiber laser. Opt. Express 25, 33216-33225 (2017).

17. Shen, X. L. et al. Electronic control of nonlinear-polarization-rotation mode locking in Yb-doped fiber lasers. Opt. Lett. 37, 3426-3428 (2012).

18. $\mathrm{Pu}, \mathrm{G}$. Q. et al. Intelligent programmable mode-locked fiber laser with a human-like algorithm. Optica 6, 362-369 (2019).

19. Bhushan, A. S., Coppinger, F. \& Jalali, B. Time-stretched analogue-to-digital conversion. Electron. Lett. 34, 839-841 (1998).

20. Mahjoubfar, A. et al. Time stretch and its applications. Nat. Photonics $\mathbf{1 1}$ 341-351 (2017).

21. Runge, A. F. J., Broderick, N. G. R. \& Erkintalo, M. Observation of soliton explosions in a passively mode-locked fiber laser. Optica 2, 36-39 (2015).

22. Liu, M. et al. Successive soliton explosions in an ultrafast fiber laser. Opt. Lett. 41, 1181-1184 (2016)

23. Herink, G. et al. Resolving the build-up of femtosecond mode-locking with single-shot spectroscopy at $90 \mathrm{MHz}$ frame rate. Nat. Photonics 10, 321-326 (2016).

24. Liu, X. M. \& Cui, Y. D. Revealing the behavior of soliton buildup in a modelocked laser. Adv. Photonics 1, 016003 (2019).

25. Liu, X. M. \& Pang, M. Revealing the buildup dynamics of harmonic mode-locking states in ultrafast lasers. Laser Photonics Rev. 13, 1800333 (2019).

26. Chen, $\mathrm{H}$. J. et al. Buildup dynamics of dissipative soliton in an ultrafast fiber laser with net-normal dispersion. Opt. Express 26, 2972-2982 (2018).

27. Herink, G. et al. Real-time spectral interferometry probes the internal dynamics of femtosecond soliton molecules. Science 356, 50-54 (2017).

28. Liu, Xm, Yao, X. K. \& Cui, Y. D. Real-time observation of the buildup of soliton molecules. Phys. Rev. Lett. 121, 023905 (2018).

29. Peng, J. S. \& Zeng, H. P. Build-Up of dissipative optical soliton molecules via diverse soliton interactions. Laser Photonics Rev. 12, 1800009 (2018).

30. Krupa, K. et al. Real-time observation of internal motion within ultrafast dissipative optical soliton molecules. Phys. Rev. Lett. 118 243901 (2017).

31. Ryczkowski, P. et al. Real-time full-field characterization of transient dissipative soliton dynamics in a mode-locked laser. Nat. Photonics 12, 221-227 (2018)

32. Sun, S. Q. et al. Time-stretch probing of ultra-fast soliton dynamics related to Q-switched instabilities in mode-locked fiber laser. Opt. Express $\mathbf{2 6}$ 20888-20901 (2018)

33. Wei, X. M. et al. Unveiling multi-scale laser dynamics through time-stretch and time-lens spectroscopies. Opt. Express 25, 29098-29120 (2017).

34. Lapre, C. et al. Real-time characterization of spectral instabilities in a modelocked fiber laser exhibiting soliton-similariton dynamics. Sci. Rep. 9, 13950 (2019).

35. Liu, X. M., Popa, D. \& Akhmediev, N. Revealing the transition dynamics from $Q$ switching to mode locking in a soliton laser. Phys. Rev. Lett. 123, 093901 (2019).

36. Pu, G. Q. et al. Real-time observation of the regime transition dynamics of mode-locked fiber lasers. IEEE Photonics Technol. Lett. 31, 1545-1548 (2019).

37. Woodward, R. I. Dispersion engineering of mode-locked fiber lasers. J. Opt. 20 033002 (2018).

38. Holland, J. H. Genetic algorithms. Sci. Am. 267 66-73 (1992)

39. Buckley, B. W., Madni, A. M. \& Jalali, B. Coherent time-stretch transformation for real-time capture of wideband signals. Opt. Express 21, 21618-21627 (2013).

40. DeVore, P. T. S. et al. Coherent time-stretch transform for near-field spectroscopy. IEEE Photonics J. 6, 3300107 (2014).

41. Mahjoubfar, A. et al. Label-free high-throughput cell screening in flow. Biomed. Opt. Express 4, 1618-1625 (2013).

42. Chen, C. L. et al. Deep learning in label-free cell classification. Sci. Rep. 6, 21471 (2016).

43. Dudley, J. M. et al. Rogue waves and analogies in optics and oceanography. Nat. Rev. Phys. 1, 675-689 (2019). 